(C) 1998 International Press

Adv. Theor. Math. Phys. 2 (1998) 231-252

\title{
The Large N Limit of Superconformal field theories and supergravity
}

\author{
Juan Maldacena ${ }^{1}$ \\ Lyman Laboratory of Physics \\ Harvard University \\ Cambridge, MA 02138, U.S.A.
}

\begin{abstract}
We show that the large $N$ limit of certain conformal field theories in various dimensions include in their Hilbert space a sector describing supergravity on the product of Anti-deSitter spacetimes, spheres and other compact manifolds. This is shown by taking some branes in the full $\mathrm{M}$ /string theory and then taking a low energy limit where the field theory on the brane decouples from the bulk. We observe that, in this limit, we can still trust the near horizon geometry for large $N$. The enhanced supersymmetries of the near horizon geometry correspond to the extra supersymmetry generators present in the superconformal group (as opposed to just the super-Poincare group). The 't Hooft limit of $3+1 \mathcal{N}=4$ super-Yang-Mills at the conformal point is shown to contain strings: they are IIB strings. We conjecture that compactifications of $\mathrm{M} /$ string theory on various Anti-deSitter spacetimes is dual to various conformal field theories. This leads to a new proposal for a definition of M-theory which could be extended to include five noncompact dimensions.
\end{abstract}

\section{General Idea}

In the last few years it has been extremely fruitful to derive quantum field theories by taking various limits of string or M-theory. In some cases this

\footnotetext{
${ }^{1}$ malda@pauli.harvard.edu
} 
is done by considering the theory at geometric singularities and in others by considering a configuration containing branes and then taking a limit where the dynamics on the brane decouples from the bulk. In this paper we consider theories that are obtained by decoupling theories on branes from gravity. We focus on conformal invariant field theories but a similar analysis could be done for non-conformal field theories. The cases considered include $N$ parallel D3 branes in IIB string theory and various others. We take the limit where the field theory on the brane decouples from the bulk. At the same time we look at the near horizon geometry and we argue that the supergravity solution can be trusted as long as $N$ is large. $N$ is kept fixed as we take the limit. The approach is similar to that used in [1] to study the NS fivebrane theory [2] at finite temperature. The supergravity solution typically reduces to $p+2$ dimensional Anti-deSitter space $\left(A d S_{p+2}\right)$ times spheres (for D3 branes we have $A d S_{5} \times S^{5}$ ). The curvature of the sphere and the $A d S$ space in Planck units is a (positive) power of $1 / N$. Therefore the solutions can be trusted as long as $N$ is large. Finite temperature configurations in the decoupled field theory correspond to black hole configurations in $A d S$ spacetimes. These black holes will Hawking radiate into the $A d S$ spacetime. We conclude that excitations of the $A d S$ spacetime are included in the Hilbert space of the corresponding conformal field theories. A theory in $A d S$ spacetime is not completely well defined since there is a horizon and it is also necessary to give some boundary conditions at infinity. However, local properties and local processes can be calculated in supergravity when $N$ is large if the proper energies involved are much bigger than the energy scale set by the cosmological constant (and smaller than the Planck scale). We will conjecture that the full quantum $\mathrm{M} /$ string-theory on $A d S$ space, plus suitable boundary conditions is dual to the corresponding brane theory. We are not going to specify the boundary conditions in $A d S$, we leave this interesting problem for the future. The $A d S \times$ (spheres) description will become useful for large $N$, where we can isolate some local processes from the question of boundary conditions. The supersymmetries of both theories agree, both are given by the superconformal group. The superconformal group has twice the amount of supersymmetries of the corresponding superPoincare group [3,4]. This enhancement of supersymmetry near the horizon of extremal black holes was observed in $[5,6]$ precisely by showing that the near throat geometry reduces to $A d S \times$ (spheres). $A d S$ spaces (and branes in them) were extensively considered in the literature [7-13], including the connection with the superconformal group.

In section 2 we study $\mathcal{N}=4 \mathrm{~d}=4 U(N)$ super-Yang-Mills as a first example, we discuss several issues which are present in all other cases. In section 3 we analyze the theories describing M-theory five-branes and M-theory twobranes. In section 4 we consider theories with lower supersymmetry which 
are related to a black string in six dimensions made with D1 and D5 branes. In section 5 we study theories with even less supersymmetry involving black strings in five dimensions and finally we mention the theories related to extremal Reissner-Nordström black holes in four spacetime dimensions (these last cases will be more speculative and contain some unresolved puzzles). Finally in section 6 we make some comments on the relation to matrix theory.

\section{D3 Branes or $\mathcal{N}=4 \quad U(N)$ Super-Yang-Mills in $d=3+1$}

We start with type IIB string theory with string coupling $g$, which will remain fixed. Consider $N$ parallel D3 branes separated by some distances which we denote by $r$. For low energies the theory on the D3 brane decouples from the bulk. It is more convenient to take the energies fixed and take

$$
\alpha^{\prime} \rightarrow 0, \quad U \equiv \frac{r}{\alpha^{\prime}}=\text { fixed } .
$$

The second condition is saying that we keep the mass of the stretched strings fixed. As we take the decoupling limit we bring the branes together but the the Higgs expectation values corresponding to this separation remains fixed. The resulting theory on the brane is four dimensional $\mathcal{N}=4 U(N)$ SYM. Let us consider the theory at the superconformal point, where $r=0$. The conformal group is $\mathrm{SO}(2,4)$. We also have an $S O(6) \sim S U(4)$ R-symmetry that rotates the six scalar fields into each other ${ }^{2}$. The superconformal group includes twice the number of supersymmetries of the super-Poincare group: the commutator of special conformal transformations with Poincare supersymmetry generators gives the new supersymmetry generators. The precise superconformal algebra was computed in [3]. All this is valid for any $N$.

Now we consider the supergravity solution carrying D3 brane charge [14]

$$
\begin{aligned}
d s^{2} & =f^{-1 / 2} d x_{\|}^{2}+f^{1 / 2}\left(d r^{2}+r^{2} d \Omega_{5}^{2}\right), \\
f & =1+\frac{4 \pi g N \alpha^{\prime 2}}{r^{4}}
\end{aligned}
$$

where $x_{\|}$denotes the four coordinates along the worldvolume of the threebrane and $d \Omega_{5}^{2}$ is the metric on the unit five-sphere ${ }^{3}$. The self dual five-form field strength is nonzero and has a flux on the five-sphere. Now we define the new variable $U \equiv \frac{r}{\alpha^{\prime}}$ and we rewrite the metric in terms of $U$. Then we

\footnotetext{
${ }^{2}$ The representation includes objects in the spinor representations, so we should be talking about SU(4), we will not make this, or similar distinctions in what follows.

${ }^{3}$ We choose conventions where $g \rightarrow 1 / g$ under S-duality.
} 
take the $\alpha^{\prime} \rightarrow 0$ limit. Notice that $U$ remains fixed. In this limit we can neglect the 1 in the harmonic function (2.2). The metric becomes

$$
d s^{2}=\alpha^{\prime}\left[\frac{U^{2}}{\sqrt{4 \pi g N}} d x_{\|}^{2}+\sqrt{4 \pi g N} \frac{d U^{2}}{U^{2}}+\sqrt{4 \pi g N} d \Omega_{5}^{2}\right] .
$$

This metric describes five dimensional Anti-deSitter $\left(A d S_{5}\right)$ times a fivesphere $^{4}$. We see that there is an overall $\alpha^{\prime}$ factor. The metric remains constant in $\alpha^{\prime}$ units. The radius of the five-sphere is $R_{s p h}^{2} / \alpha^{\prime}=\sqrt{4 \pi g N}$, and is the same as the "radius" of $A d S_{5}$ (as defined in the appendix). In ten dimensional Planck units they are both proportional to $N^{1 / 4}$. The radius is quantized because the flux of the 5 -form field strength on the 5 sphere is quantized. We can trust the supergravity solution when

$$
g N \gg 1 \text {. }
$$

When $N$ is large we have approximately ten dimensional flat space in the neighborhood of any point ${ }^{5}$. Note that in the large $N$ limit the flux of the 5 form field strength per unit Planck (or string) 5 -volume becomes small.

Now consider a near extremal black D3 brane solution in the decoupling limit (2.1). We keep the energy density on the brane worldvolume theory $(\mu)$ fixed. We find the metric

$$
\begin{aligned}
d s^{2}= & \alpha^{\prime}\left\{\frac{U^{2}}{\sqrt{4 \pi g N}}\left[-\left(1-U_{0}^{4} / U^{4}\right) d t^{2}+d x_{i}^{2}\right]\right. \\
& \left.+\sqrt{4 \pi g N} \frac{d U^{2}}{U^{2}\left(1-U_{0}^{4} / U^{4}\right)}+\sqrt{4 \pi g N} d \Omega_{5}^{2}\right\} . \\
U_{0}^{4}= & \frac{2^{7}}{3} \pi^{4} g^{2} \mu
\end{aligned}
$$

We see that $U_{0}$ remains finite when we take the $\alpha^{\prime} \rightarrow 0$ limit. The situation is similar to that encountered in [1]. Naively the whole metric is becoming of zero size since we have a power of $\alpha^{\prime}$ in front of the metric, and we might incorrectly conclude that we should only consider the zero modes of all fields. However, energies that are finite from the point of view of the gauge theory, lead to proper energies (measured with respect to proper time) that remain finite is in $\alpha^{\prime}$ units (or Planck units, since $g$ is fixed). More concretely, an excitation that has energy $\omega$ (fixed in the limit) from the point of view of the gauge theory, will have proper energy $E_{\text {proper }}=\frac{1}{\sqrt{\alpha^{\prime}}} \frac{\omega(g N 4 \pi)^{1 / 4}}{U}$. This also means that the corresponding proper wavelengths remain fixed. In

\footnotetext{
${ }^{4}$ See the appendix for a brief description of $A d S$ spacetimes.

${ }^{5}$ In writing (2.4) we assumed that $g \leq 1$, if $g>1$ then the condition is $N / g \gg 1$. In other words we need large $N$, not large $g$.
} 
other words, the spacetime action on this background has the form $S \sim$ $\frac{1}{\alpha^{\prime 4}} \int d^{10} x \sqrt{G} R+\cdots$, so we can cancel the factor of $\alpha^{\prime}$ in the metric and the Newton constant, leaving a theory with a finite Planck length in the limit. Therefore we should consider fields that propagate on the $A d S$ background. Since the Hawking temperature is finite, there is a flux of energy from the black hole to the $A d S$ spacetime. Since $\mathcal{N}=4 \mathrm{~d}=4 U(N)$ SYM is a unitary theory we conclude that, for large $N$, it includes in its Hilbert space the states of type IIB supergravity on $\left(\operatorname{Ad} S_{5} \times S_{5}\right)_{N}$, where subscript indicates the fact that the "radii" in Planck units are proportional to $N^{1 / 4}$. In particular the theory contains gravitons propagating on $\left(A d S_{5} \times S_{5}\right)_{N}$. When we consider supergravity on $A d S_{5} \times S_{5}$, we are faced with global issues like the presence of a horizon and the boundary conditions at infinity. It is interesting to note that the solution is nonsingular [15]. The gauge theory should provide us with a specific choice of boundary conditions. It would be interesting to determine them.

We have started with a quantum theory and we have seen that it includes gravity so it is natural to think that this correspondence goes beyond the supergravity approximation. We are led to the conjecture that Type IIB string theory on $\left(A d S_{5} \times S^{5}\right)_{N}$ plus some appropriate boundary conditions (and possibly also some boundary degrees of freedom) is dual to $N=4 d=3+1$ $U(N)$ super-Yang-Mills. The SYM coupling is given by the (complex) IIB string coupling, more precisely $\frac{1}{g_{Y M}^{2}}+i \frac{\theta}{8 \pi^{2}}=\frac{1}{2 \pi}\left(\frac{1}{g}+i \frac{\chi}{2 \pi}\right)$ where $\chi$ is the value of the $R R$ scalar.

The supersymmetry group of $A d S_{5} \times S^{5}$, is known to be the same as the superconformal group in $3+1$ spacetime dimensions [3], so the supersymmetries of both theories are the same. This is a new form of "duality": a large $N$ field theory is related to a string theory on some background, notice that the correspondence is non-perturbative in $g$ and the $S L(2, Z)$ symmetry of type IIB would follow as a consequence of the $S L(2, Z)$ symmetry of $\mathrm{SYM}^{6}$. It is also a strong-weak coupling correspondence in the following sense. When the effective coupling $g N$ becomes large we cannot trust perturbative calculations in the Yang-Mills theory but we can trust calculations in supergravity on $\left(A d S_{5} \times S^{5}\right)_{N}$. This is suggesting that the $\mathcal{N}=4$ Yang-Mills master field is the anti-deSitter supergravity solution (similar ideas were suggested in [17]). Since $N$ measures the size of the geometry in Planck units, we see that quantum effects in $A d S_{5} \times S^{5}$ have the interpretation of $1 / N$ effects in the gauge theory. So Hawking radiation is a $1 / N$ effect. It would be interesting to understand more precisely what the horizon means from the gauge theory point of view. IIB supergravity on $A d S_{5} \times S^{5}$ was studied in [7,9].

The above conjecture becomes nontrivial for large $N$ and gives a way to answer some large $N$ questions in the SYM theory. For example, suppose

\footnotetext{
${ }^{6}$ This is similar in spirit to [16] but here $N$ is not interpreted as momentum.
} 
that we break $U(N) \rightarrow U(N-1) \times U(1)$ by Higgsing. This corresponds to putting a three brane at some point on the 5 -sphere and some value of $U$, with world volume directions along the original four dimensions $\left(x_{\|}\right)$. We could now ask what the low energy effective action for the light U(1) fields is. For large $N(2.4)$ it is the action of a D3 brane in $A d S_{5} \times S^{5}$. More concretely, the bosonic part of the action becomes the Born-Infeld action on the $A d S$ background

$$
\begin{aligned}
S & =-\frac{1}{(2 \pi)^{3} g} \int d^{4} x h^{-1} \\
& \cdot\left[\sqrt{-\operatorname{Det}\left(\eta_{\alpha \beta}+h \partial_{\alpha} U \partial_{\beta} U+U^{2} h g_{i j} \partial_{\alpha} \theta^{i} \partial_{\beta} \theta^{j}+2 \pi \sqrt{h} F_{\alpha \beta}\right)}-1\right] \\
h & =\frac{4 \pi g N}{U^{4}},
\end{aligned}
$$

with $\alpha, \beta=0,1,2,3, i, j=1, . ., 5$; and $g_{i j}$ is the metric of the unit fivesphere. As any low energy action, (2.6) is valid when the energies are low compared to the mass of the massive states that we are integrating out. In this case the mass of the massive states is proportional to $U$ (with no factors of $N)$. The low energy condition translates into $\partial U / U \ll U$ and $\partial \theta^{i}<<U$, etc.. So the nonlinear terms in the action (2.6) will be important only when $g N$ is large. It seems that the form of this action is completely determined by superconformal invariance, by using the broken and unbroken supersymmetries, in the same sense that the Born Infeld action in flat space is given by the full Poincare supersymmetry [18]. It would be very interesting to check this explicitly. We will show this for a particular term in the action. We set $\theta^{i}=$ const and $F=0$, so that we only have $U$ left. Then we will show that the action is completely determined by broken conformal invariance. This can be seen as follows. Using Lorentz invariance and scaling symmetry (dimensional analysis) one can show that the action must have the form

$$
S=\int d^{p+1} x U^{p+1} f\left(\partial_{\alpha} U \partial^{\alpha} U / U^{4}\right)
$$

where $f$ is an arbitrary function. Now we consider infinitesimal special conformal transformations

$$
\begin{aligned}
\delta x^{\alpha} & =\epsilon^{\beta} x_{\beta} x^{\alpha}-\epsilon^{\alpha}\left(x^{2}+\frac{\tilde{R}^{4}}{U^{2}}\right) / 2, \\
\delta U & \equiv U^{\prime}\left(x^{\prime}\right)-U(x)=-\epsilon^{\alpha} x_{\alpha} U,
\end{aligned}
$$

where $\epsilon^{\alpha}$ is an infinitesimal parameter. For the moment $\tilde{R}$ is an arbitrary constant. We will later identify it with the "radius" of $A d S$, it will turn out 
that $\tilde{R}^{4} \sim g N$. In the limit of small $\tilde{R}$ we recover the more familiar form of the conformal transformations ( $U$ is a weight one field). Usually conformal transformations do not involve the variable $U$ in the transformations of $x$. For constant $U$ the extra term in (2.8) is a translation in $x$, but we will take $U$ to be a slowly varying function of $x$ and we will determine $\tilde{R}$ from other facts that we know. Demanding that (2.7) is invariant under (2.8) we find that the function $f$ in (2.7) obeys the equation

$$
f(z)+\text { const }=2\left(z+\frac{1}{\tilde{R}^{4}}\right) f^{\prime}(z)
$$

which is solved by $f=b\left[\sqrt{1+\tilde{R}^{4} z}-a\right]$. Now we can determine the constants $a, b, \tilde{R}$ from supersymmetry. We need to use three facts. The first is that there is no force (no vacuum energy) for a constant $U$. This implies $a=$ 1. The second is that the $\partial U^{2}$ term $\left(F^{2}\right.$ term) in the $U(1)$ action is not renormalized. The third is that the only contribution to the $(\partial U)^{4}$ term (an $F^{4}$ term) comes from a one loop diagram [19]. This determines all the coefficients to be those expected from (2.6) including the fact that $\tilde{R}^{4}=$ $4 \pi g N$. It seems very plausible that using all 32 supersymmetries we could fix the action (2.6) completely. This would be saying that (2.6) is a consequence of the symmetries and thus not a prediction ${ }^{7}$. However we can make very nontrivial predictions (though we were not able to check them). For example, if we take $g$ to be small (but $N$ large) we can predict that the Yang-Mills theory contains strings. More precisely, in the limit $g \rightarrow 0, g N=$ fixed $\gg 1$ ('t Hooft limit) we find free strings in the spectrum, they are IIB strings moving in $\left(A d S_{5} \times S^{5}\right)_{g N} .{ }^{8}$ The sense in which these strings are present is rather subtle since there is no energy scale in the Yang-Mills to set their tension. In fact one should translate the mass of a string state from the $A d S$ description to the Yang-Mills description. This translation will involve the position $U$ at which the string is sitting. This sets the scale for its mass. As an example, consider again the D-brane probe (Higgsed configuration) which we described above. From the type IIB description we expect open strings ending on the D3 brane probe. From the point of view of the gauge theory these open strings have energies $E=\frac{U}{(4 \pi g N)^{1 / 4}} \sqrt{N_{\text {open }}}$ where $N_{\text {open }}$ is the integer charaterizing the massive open string level. In this example we

\footnotetext{
${ }^{7}$ Notice that the action (2.6) includes a term proportional to $v^{6}$ similar to that calculated in [20]. Conformal symmetry explains the agreement that they would have found if they had done the calculation for $3+1$ SYM as opposed to $0+1$.

${ }^{8}$ In fact, Polyakov [21] recently proposed that the string theory describing bosonic YangMills has a new dimension corresponding to the Liouville mode $\varphi$, and that the metric at $\varphi=0$ is zero due to a "zig-zag" symmetry. In our case we see that the physical distances along the directions of the brane contract to zero as $U \rightarrow 0$. The details are different, since we are considering the $\mathcal{N}=4$ theory.
} 
see that $\alpha^{\prime}$ disappears when we translate the energies and is replaced by $U$, which is the energy scale of the Higgs field that is breaking the symmetry.

Now we turn to the question of the physical interpretation of $U . U$ has dimensions of mass. It seems natural to interpret motion in $U$ as moving in energy scales, going to the IR for small $U$ and to the UV for large U. For example, consider a D3 brane sitting at some position $U$. Due to the conformal symmetry, all physics at energy scales $\omega$ in this theory is the same as physics at energies $\omega^{\prime}=\lambda \omega$, with the brane sitting at $U^{\prime}=\lambda U$.

Now let us turn to another question. We could separate a group of D3 branes from the point were they were all sitting originally. Fortunately, for the extremal case we can find a supergravity solution describing this system. All we have to do is the replacement

$$
\frac{N}{U^{4}} \rightarrow \frac{N-M}{U^{4}}+\frac{M}{|\vec{U}-\vec{W}|^{4}},
$$

where $\vec{W}=\vec{r} / \alpha^{\prime}$ is the separation. It is a vector because we have to specify a point on $S^{5}$ also. The resulting metric is

$$
\begin{aligned}
& d s^{2}=\alpha^{\prime}\left[U^{2} \frac{1}{\sqrt{4 \pi g}\left(N-M+\frac{M U^{4}}{|\vec{U}-W|^{4}}\right)^{1 / 2}} d x_{\|}^{2}\right. \\
& \left.+\sqrt{4 \pi g} \frac{1}{U^{2}}\left(N-M+\frac{M U^{4}}{|\vec{U}-W|^{4}}\right)^{1 / 2} d \vec{U}^{2}\right] .
\end{aligned}
$$

For large $U \gg|W|$ we find basically the solution for $\left(A d S_{5} \times S^{5}\right)_{N}$ which is interpreted as saying that for large energies we do not see the fact that the conformal symmetry was broken, while for small $U \ll|W|$ we find just $\left(A d S_{5} \times S^{5}\right)_{N-M}$, which is associated to the CFT of the unbroken $U(N-M)$ piece. Furthermore, if we consider the region $|\vec{U}-\vec{W}| \ll|\vec{W}|$ we find $\left(A d S_{5} \times S^{5}\right)_{M}$, which is described by the CFT of the $U(M)$ piece.

We could in principle separate all the branes from each other. For large values of $U$ we would still have $\left(A d S_{5} \times S^{5}\right)_{N}$, but for small values of $U$ we would not be able to trust the supergravity solution, but we naively get $N$ copies of $\left(A d S_{5} \times S^{5}\right)_{1}$ which should correspond to the $U(1)^{N}$.

Now we discuss the issue of compactification. We want to consider the YM theory compactified on a torus of radii $R_{i}, x_{i} \sim x_{i}+2 \pi R_{i}$, which stay fixed as we take the $\alpha^{\prime} \rightarrow 0$ limit. Compactifying the theory breaks conformal invariance and leaves only the Poincare supersymmetries. However one can still find the supergravity solutions and follow the above procedure, going near the horizon, etc. The $A d S$ piece will contain some identifications. So 
we will be able to trust the supergravity solution as long as the physical length of these compact circles stays big in $\alpha^{\prime}$ units. This implies that we can trust the supergravity solution as long as we stay far from the horizon (at $U=0$ )

$$
U \gg \frac{(g N)^{1 / 4}}{R_{i}},
$$

for all $i$. This is a larger bound than the naive expectation $\left(1 / R_{i}\right)$. If we were considering near extremal black holes we would require that $U_{0}$ in (2.5) satisfies (2.12), which is, of course, the same condition on the temperature gotten in [22].

The relation of the three-brane supergravity solution and the Yang-Mills theory has been studied in [23-26]. All the calculations have been done for near extremal D3 branes fall into the category described above. In particular the absorption cross section of the dilaton and the graviton have been shown to agree with what one would calculate in the YM theory [24,25]. It has been shown in [26] that some of these agreements are due to non-renormalization theorems for $\mathcal{N}=4 \mathrm{YM}$. The black hole entropy was compared to the perturbative YM calculation and it agrees up to a numerical factor [23]. This is not in disagreement with the correspondence we were suggesting, It is expected that large $g N$ effects change this numerical factor, this problem remains unsolved.

Finally notice that the group $S O(2,4) \times S O(6)$ suggests a twelve dimensional realization in a theory with two times [27].

\section{Other Cases with $16 \rightarrow 32$ Supersymmetries, M5 and M2 Brane Theories}

Basically all that we have said for the D3 brane carries over for the other conformal field theories describing coincident M-theory fivebranes and Mtheory twobranes. We describe below the limits that should be taken in each of the two cases. Similar remarks can be made about the entropies [28], and the determination of the probe actions using superconformal invariance. Eleven dimensional supergravity on the corresponding $A d S$ spaces was studied in $[8,10,11,15]$.

\subsection{M5 Brane}

The decoupling limit is obtained by taking the 11 dimensional Planck length to zero, $l_{p} \rightarrow 0$, keeping the worldvolume energies fixed and taking the separations $U^{2} \equiv r / l_{p}^{3}=$ fixed [29]. This last condition ensures that the 
membranes stretching between fivebranes give rise to strings with finite tension.

The metric is ${ }^{9}$

$$
\begin{aligned}
d s^{2} & =f^{-1 / 3} d x_{\|}^{2}+f^{2 / 3}\left(d r^{2}+r^{2} d \Omega_{4}^{2}\right), \\
f & =1+\frac{\pi N l_{p}^{3}}{r^{3}}
\end{aligned}
$$

We also have a flux of the four-form field strength on the four-sphere (which is quantized). Again, in the limit we obtain

$$
d s^{2}=l_{p}^{2}\left[\frac{U^{2}}{(\pi N)^{1 / 3}} d x_{\|}^{2}+4(\pi N)^{2 / 3} \frac{d U^{2}}{U^{2}}+(\pi N)^{2 / 3} d \Omega_{4}^{2}\right],
$$

where now the "radii" of the sphere and the $A d S_{7}$ space are $R_{s p h}=$ $R_{A d S} / 2=l_{p}(\pi N)^{1 / 3}$. Again, the "radii" are fixed in Planck units as we take $l_{p} \rightarrow 0$, and supergravity can be applied if $N \gg 1$.

Reasoning as above we conclude that this theory contains seven dimensional Anti-deSitter times a four-sphere, which for large $N$ looks locally like eleven dimensional Minkowski space.

This gives us a method to calculate properties of the large $\mathrm{N}$ limit of the six dimensional $(0,2)$ conformal field theory [30]. The superconformal group again coincides with the algebra of the supersymmetries preserved by $A d S_{7} \times S^{4}$. The bosonic symmetries are $S O(2,6) \times S O(5)$ [4]. We can do brane probe calculations, thermodynamic calculations [28], etc.

The conjecture is now that the $(0,2)$ conformal field theory is dual to $M$-theory on $\left(A d S_{7} \times S^{4}\right)_{N}$, the subindex indicates the dependence of the "radius" with $N$.

\subsection{M2 Brane}

We now take the limit $l_{p} \rightarrow 0$ keeping $U^{1 / 2} \equiv r / l_{p}^{3 / 2}=$ fixed. This combination has to remain fixed because the scalar field describing the motion of the twobrane has scaling dimension $1 / 2$. Alternatively we could have derived this conformal field theory by taking first the field theory limit of D2 branes in string theory as in [31-33], and then taking the strong coupling limit of that theory to get to the conformal point as in [34-36]. The fact that the theories obtained in this fashion are the same can be seen as follows. The D2 brane gauge theory can be obtained as the limit $\alpha^{\prime} \rightarrow 0$, keeping $g_{Y M}^{2} \sim g / \alpha^{\prime}=$ fixed. This is the same as the limit of M-theory two branes in the limit $l_{p} \rightarrow 0$ with $R_{11} / l_{p}^{3 / 2} \sim g_{Y M}=$ fixed. This is a theory where

\footnotetext{
${ }^{9}$ In our conventions the relation of the Planck length to the 11 dimensional Newton constant is $G_{N}^{11}=16 \pi^{7} l_{p}^{9}$.
} 
one of the Higgs fields is compact. Taking $R^{11} \rightarrow \infty$ we see that we get the theory of coincident $\mathrm{M} 2$ branes, in which the $\mathrm{SO}(8) \mathrm{R}$-symmetry has an obvious origin.

The metric is

$$
\begin{aligned}
d s^{2} & =f^{-2 / 3} d x_{\|}^{2}+f^{1 / 3}\left(d r^{2}+r^{2} d \Omega_{7}^{2}\right), \\
f & =1+\frac{2^{5} \pi^{2} N l_{p}^{6}}{r^{6}}
\end{aligned}
$$

and there is a nonzero flux of the dual of the four-form field strength on the seven-sphere. In the decoupling limit we obtain $A d S_{4} \times S^{7}$, and the supersymmetries work out correctly. The bosonic generators are given by $S O(2,3) \times S O(8)$. In this case the "radii" of the sphere and $A d S_{4}$ are $R_{s p h}=2 R_{A d S}=l_{p}\left(2^{5} \pi^{2} N\right)^{1 / 6}$.

The entropy of the near extremal solution agrees with the expectation from dimensional analysis for a conformal theory in 2+1 dimensions [28], but the $N$ dependence or the numerical coefficients are not understood.

Actually for the case of the two brane the conformal symmetry was used to determine the $v^{4}$ term in the probe action [37], we are further saying that conformal invariance determines it to all orders in the velocity of the probe. Furthermore the duality we have proposed with M-theory on $A d S_{4} \times S^{7}$ determines the precise numerical coefficient.

When M-theory is involved the dimensionalities of the groups are suggestive of a thirteen dimensional realization [38].

\section{Theories with $8 \rightarrow 16$ Supersymmetries, the D1+D5 System}

Now we consider IIB string theory compactified on $M^{4}$ (where $M^{4}=T^{4}$ or $K 3)$ to six spacetime dimensions. As a first example let us start with a D-fivebrane with four dimensions wrapping on $M^{4}$ giving a string in six dimensions. Consider a system with $Q_{5}$ fivebranes and $Q_{1} \mathrm{D}$-strings, where the $\mathrm{D}$-string is parallel to the string in six dimensions arising from the fivebrane. This system is described at low energies by a $1+1$ dimensional $(4,4)$ superconformal field theory. So we take the limit

$$
\alpha^{\prime} \rightarrow 0, \quad \frac{r}{\alpha^{\prime}}=\text { fixed }, \quad v \equiv \frac{V_{4}}{(2 \pi)^{4} \alpha^{2}}=\text { fixed }, \quad g_{6}=\frac{g}{\sqrt{v}}=\text { fixed }
$$

where $V_{4}$ is the volume of $M^{4}$. All other moduli of $M^{4}$ remain fixed. This is just a low energy limit, we keep all dimensionless moduli fixed. As a six dimensional theory, IIB on $M^{4}$ contains strings. They transform under the 
U-duality group and they carry charges given by a vector $q^{I}$. In general we can consider a configuration where $q^{2}=\eta_{I J} q^{I} q^{J} \neq 0$ (the metric is the U-duality group invariant), and then take the limit (4.1).

This theory has a branch which we will call the Higgs branch and one which we call the Coulomb branch. On the Higgs branch the 1+1 dimensional vector multiplets have zero expectation value and the Coulomb branch is the other one. Notice that the expectation values of the vector multiplets in the Coulomb branch remain fixed as we take the limit $\alpha^{\prime} \rightarrow 0$.

The Higgs branch is a SCFT with $(4,4)$ supersymmetry. This is the theory considered in [39]. The above limit includes also a piece of the Coulomb branch, since we can separate the branes by a distance such that the mass of stretched strings remains finite.

Now we consider the supergravity solution corresponding to D1+D5 branes [40]

$$
\begin{aligned}
d s^{2} & =f_{1}^{-1 / 2} f_{5}^{-1 / 2} d x_{\|}^{2}+f_{1}^{1 / 2} f_{5}^{1 / 2}\left(d r^{2}+r^{2} d \Omega_{3}^{2}\right), \\
f_{1} & =\left(1+\frac{g \alpha^{\prime} Q_{1}}{v r^{2}}\right), \quad f_{5}=\left(1+\frac{g \alpha^{\prime} Q_{5}}{r^{2}}\right),
\end{aligned}
$$

where $d x_{\|}^{2}=-d t^{2}+d x^{2}$ and $x$ is the coordinate along the D-string. Some of the moduli of $M^{4}$ vary over the solution and attain a fixed value at the horizon which depends only on the charges and some others are constant throughout the solution. The three-form RR-field strength is also nonzero.

In the decoupling limit (4.1) we can neglect the 1 's in $f_{i}$ in (4.2) and the metric becomes

$$
d s^{2}=\alpha^{\prime}\left[\frac{U^{2}}{g_{6} \sqrt{Q_{1} Q_{5}}} d x_{\|}^{2}+g_{6} \sqrt{Q_{1} Q_{5}} \frac{d U^{2}}{U^{2}}+g_{6} \sqrt{Q_{1} Q_{5}} d \Omega_{3}^{2}\right] .
$$

The compact manifold $M^{4}(Q)$ that results in the limit is determined as follows. Some of its moduli are at their fixed point value which depends only on the charges and not on the asymptotic value of those moduli at infinity (the notation $M^{4}(Q)$ indicates the charge dependence of the moduli) [41] ${ }^{10}$. The other moduli, that were constant in the black hole solution, have their original values. For example, the volume of $M^{4}$ has the fixed point value $v_{\text {fixed }}=Q_{1} / Q_{5}$, while the six dimensional string coupling $g_{6}$ has the original value. Notice that there is an overall factor of $\alpha^{\prime}$ in (4.3) which can be removed by canceling it with the factor of $\alpha^{\prime}$ in the Newton constant as explained above. We can trust the supergravity solution if $Q_{1}, Q_{5}$ are large, $g_{6} Q_{i} \gg 1$. Notice that we are talking about a six dimensional supergravity

\footnotetext{
${ }^{10}$ The fixed values of the moduli are determined by the condition that they minimize the tension of the corresponding string (carrying charges $q^{I}$ ) in six dimensions [41]. This is parallel to the discussion in four dimensions [42].
} 
solution since the volume of $M^{4}$ is a constant in Planck units (we keep the $Q_{1} / Q_{5}$ ratio fixed). The metric (4.3) describes three dimensional $A d S_{3}$ times a 3 -sphere. The supersymmetries work out correctly, starting from the 8 Poincare supersymmetries we enhance then to 16 supersymmetries. The bosonic component is $S O(2,2) \times S O(4)$. In conformal field theory language $S O(2,2)$ is just the $S L(2, R) \times S L(2, R)$ part of the conformal group and $\mathrm{SO}(4) \sim S U(2)_{L} \times S U(2)_{R}$ are the R-symmetries of the CFT [43].

So the conjecture is that the $1+1$ dimensional CFT describing the Higgs branch of the D1+D5 system on $M^{4}$ is dual to type IIB string theory on

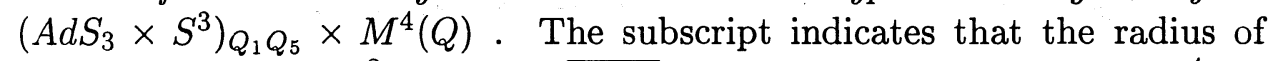
the three sphere is $R_{s p h}^{2}=\alpha^{\prime} g_{6} \sqrt{Q_{1} Q_{5}}$. The compact fourmanifold $M^{4}(Q)$ is at some particular point in moduli space determined as follows. The various moduli of $M^{4}$ are divided as tensors and hypers according to the $(4,4)$ supersymmetry on the brane. Each hypermultiplet contains four moduli and each tensor contains a modulus and an anti-self-dual $B$-field. (There are five tensors of this type for $T^{4}$ and 21 for $K 3$ ). The scalars in the tensors have fixed point values at the horizon of the black hole, and those values are the ones entering in the definition of $M^{4}(Q)$ ( $Q$ indicates the dependence on the charges). The hypers will have the same expectation value everywhere. It is necessary for this conjecture to work that the $1+1$ dimensional $(4,4)$ theory is indendent of the tensor moduli appearing in its original definition as a limit of the brane configurations, since $M^{4}(Q)$ does not depend on those moduli. A non renomalization theorem like $[44,45]$ would explain this. We also need that the Higgs branch decouples from the Coulomb branch as in $[46,47]$.

Finite temperature configurations in the $1+1$ conformal field theory can be considered. They correspond to near extremal black holes in $A d S_{3}$. The metric is the same as that of the BTZ $2+1$ dimensional black hole [48], except that the angle of the BTZ description is not periodic. This angle corresponds to the spatial direction $x$ of the $1+1$ dimensional CFT and it becomes periodic if we compactify the theory ${ }^{11}[49-51]^{12}$. All calculations done for the $1 \mathrm{D}+5 \mathrm{D}$ system $[39,53,54]$ are evidence for this conjecture. In all these cases [54] the nontrivial part of the greybody factors comes from the $A d S$ part of the spacetime. Indeed, it was noticed in [55] that the greybody factors for the BTZ black hole were the same as the ones for the five-dimensional black hole in the dilute gas approximation. The

\footnotetext{
${ }^{11}$ I thank G. Horowitz for many discussions on this correspondence and for pointing out ref. [49] to me. Some of the remarks the remarks below arose in conversations with him.

${ }^{12}$ The ideas in [49-51] could be used to show the relation between the $A d S$ region and black holes in M-theory on a light like circle. However the statement in [49-51] that the $A d S_{3} \times S^{3}$ spacetime is $\mathrm{U}$-dual to the full black hole solution (which is asymptotic to Minkowski space) should be taken with caution because in those cases the spacetime has identifications on circles that are becoming null. This changes dramatically the physics. For examples of these changes see [32,52].
} 
dilute gas condition $r_{0}, r_{n} \ll r_{1} r_{5}$ [53] is automatically satisfied in the limit (4.1) for finite temperature configurations (and finite chemical potential for the momentum along $\hat{x}$ ). It was also noticed that the equations have an $S L(2, R) \times S L(2, R)$ symmetry [56], these are the isometries of $A d S_{3}$, and part of the conformal symmetry of the $1+1$ dimensional field theory. It would be interesting to understand what is the gravitational counterpart of the full conformal symmetry group in $1+1$ dimensions.

\section{Theories with $4 \rightarrow 8$ Supersymmetries}

The theories of this type will be related to black strings in five dimensions and Reissner-Nordström black holes in four dimensions. This part will be more sketchy, since there are several details of the conformal field theories involved which I do not completely understand, most notably the dependence on the various moduli of the compactification.

\subsection{Black String in Five Dimensions}

One can think about this case as arising from M-theory on $M^{6}$ where $M^{6}$ is a CY manifold, $K 3 \times T^{2}$ or $T^{6}$. We wrap fivebranes on a four-cycle $P_{4}=p^{A} \alpha_{A}$ in $M^{6}$ with nonzero triple self intersection number, see [57]. We are left with a one dimensional object in five spacetime dimensions. Now we take the following limit

$$
l_{p} \rightarrow 0 \quad(2 \pi)^{6} v \equiv V_{6} / l_{p}^{6}=\text { fixed } \quad U^{2} \equiv r / l_{p}^{3}=\text { fixed },
$$

where $l_{p}$ is the eleven dimensional Planck length. In this limit the theory will reduce to a conformal field theory in two dimensions. It is a $(0,4) \mathrm{CFT}$ and it was discussed in some detail in a region of the moduli space in [57]. More generally we should think that the five dimensional theory has some strings characterized by charges $p^{A}$, forming a multiplet of the U-duality group and we are taking a configuration where the triple self intersection number $p^{3}$ is nonzero (in the case $M^{6}=T^{6}, p^{3} \equiv D \equiv D_{A B C} p^{A} p^{B} p^{C}$ is the cubic $E_{6}$ invariant).

We now take the corresponding limit of the black hole solution. We will just present the near horizon geometry, obtained after taking the limit. Near the horizon all the vector moduli are at their fixed point values [58]. So the near horizon geometry can be calculated by considering the solution with constant moduli. We get

$$
d s^{2}=l_{p}^{2}\left[\frac{U^{2} v^{1 / 3}}{D^{1 / 3}}\left(-d t^{2}+d x^{2}\right)+\frac{D^{2 / 3}}{v^{2 / 3}}\left(4 \frac{d U^{2}}{U^{2}}+d \Omega_{2}^{2}\right)\right] .
$$


In this limit $M^{6}$ has its vector moduli equal to their fixed point values which depend only on the charge while its hyper moduli are what they were at infinity. The overall size of $M^{6}$ in Planck units is a hypermultiplet, so it remains constant as we take the limit (5.1). We get a product of three dimensional $A d S_{3}$ spacetime with a two-sphere, $A d S_{3} \times S^{2}$. Defining the five dimensional Planck length by $l_{5 p}^{3}=l_{p}^{3} / v$ we find that the "radii" of the two sphere and the $A d S_{3}$ are $R_{s p h}=R_{A d S} / 2=l_{5 p} D^{1 / 3}$. In this case the superconformal group contains as a bosonic subgroup $S O(2,2) \times S O(3)$. So the R-symmetries are just $S U(2)_{R}$, associated to the 4 rightmoving supersymmetries.

In this case we conjecture that this $(0,4)$ conformal field theory is dual, for large $p^{A}$, to M-theory on $A d S_{3} \times S^{2} \times M_{p}^{6}$. The hypermultiplet moduli of $M_{p}^{6}$ are the same as the ones entering the definition of the $(0,4)$ theory. The vector moduli depend only on the charges and their values are those that the black string has at the horizon. A necessary condition for this conjecture to work is that the $(0,4)$ theory should be independent of the original values of the vector moduli (at least for large $p$ ). It is not clear to me whether this is true.

Using this conjecture we would get for large $N$ a compactification of $M$ theory which has five extended dimensions.

\subsection{Extremal 3+1 Dimensional Reissner-Nordström}

This section is more sketchy and contains an unresolved puzzle, so the reader will not miss much if he skips it.

We start with IIB string theory compactified on $M^{6}$, where $M^{6}$ is a Calabi-Yau manifold or $K 3 \times T^{2}$ or $T^{6}$. We consider a configuration of $D 3$ branes that leads to a black hole with nonzero horizon area. Consider the limit

$$
\alpha^{\prime} \rightarrow 0 \quad(2 \pi)^{6} v \equiv \frac{V_{6}}{\alpha^{\prime 3}}=\text { fixed } \quad U \equiv \frac{r}{\alpha^{\prime}}=\text { fixed } .
$$

The string coupling is arbitrary. In this limit the system reduces to quantum mechanics on the moduli space of the three-brane configuration.

Taking the limit (5.3) of the supergravity solution we find

$$
d s^{2}=\alpha^{\prime}\left[\frac{U^{2}}{g_{4}^{2} N^{2}} d t^{2}+g_{4}^{2} N^{2} \frac{d U^{2}}{U^{2}}+g_{4}^{2} N^{2} d \Omega_{2}^{2}\right]
$$

where $N$ is proportional to the number of D3 branes. We find a two dimensional $A d S_{2}$ space times a two-sphere, both with the same radius $R=l_{4 p} N$, where $l_{4 p}^{2}=g^{2} \alpha^{\prime} / v$. The bosonic symmetries of $A d S_{2} \times S^{2}$ are $S O(2,1) \times S O(3)$. This superconformal symmetry seems related to the symmetries of the chiral conformal field theory that was proposed in [59] to 
describe the Reissner-Nordström black holes. Here we find a puzzle, since in the limit (5.3) we got a quantum mechanical system and not a $1+1$ dimensional conformal field theory. In the limit (5.3) the energy gap (mentioned in $[59,60])$ becomes very large ${ }^{13}$. So it looks like taking a large $N$ limit at the same time will be crucial in this case. These problems might be related to the large ground state entropy of the system.

If this is understood it might lead to a proposal for a non perturbative definition of $\mathrm{M} /$ string theory (as a large $N$ limit) when there are four noncompact dimensions.

It is interesting to consider the motion of probes on the $A d S_{2}$ background. This corresponds to going into the "Coulomb" branch of the quantum mechanics. Dimensional analysis says that the action has the form (2.7) with $p=0$. Expanding $f$ to first order we find $S \sim \int d t \frac{\dot{U}^{2}}{U^{3}} \sim \int d t v^{2} / r^{3}$, which is the dependence on $r$ that we expect from supergravity when we are close to the horizon. A similar analysis for Reissner-Nordström black holes in five dimensions would give a term proportional to $1 / r^{4}$ [17]. It will be interesting to check the coefficient (note that this is the only term allowed by the symmetries, as opposed to [17]).

\section{Discussion, Relation to Matrix Theory}

By deriving various field theories from string theory and considering their large $N$ limit we have shown that they contain in their Hilbert space excitations describing supergravity on various spacetimes. We further conjectured that the field theories are dual to the full quantum $\mathrm{M} /$ string theory on various spacetimes. In principle, we can use this duality to give a definition of $\mathrm{M} /$ string theory on flat spacetime as (a region of) the large $N$ limit of the field theories. Notice that this is a non-perturbative proposal for defining such theories, since the corresponding field theories can, in principle, be defined non-perturbatively. We are only scratching the surface and there are many things to be worked out. In [61] it has been proposed that the large $N$ limit of D0 brane quantum mechanics would describe eleven dimensional M-theory. The large $N$ limits discussed above, also provide a definition of M-theory. An obvious difference with the matrix model of [61] is that here $N$ is not interpreted as the momentum along a compact direction. In our case, $N$ is related to the curvature and the size of the space where the theory is defined. In both cases, in the large $N$ limit we expect to get flat, non-compact spaces. The matrix model [61] gives us a prescription to build asymptotic states, we have not shown here how to construct graviton states,

\footnotetext{
${ }^{13}$ I thank A. Strominger for pointing this out to me.
} 
and this is a very interesting problem. On the other hand, with the present proposal it is more clear that we recover supergravity in the large $N$ limit.

This approach leads to proposals involving five (and maybe in some future four) non-compact dimensions. The five dimensional proposal involves considering the $1+1$ dimensional field theory associated to a black string in five dimensions. These theories need to be studied in much more detail than we have done here.

It seems that this correspondence between the large $N$ limit of field theories and supergravity can be extended to non-conformal field theories. An example was considered in [1], where the theory of NS fivebranes was studied in the $g \rightarrow 0$ limit. A natural interpretation for the throat region is that it is a region in the Hilbert space of a six dimensional "string" theory ${ }^{14}$. And the fact that contains gravity in the large $\mathrm{N}$ limit is just a common feature of the large $N$ limit of various field theories. The large $N$ master field seems to be the anti-deSitter supergravity solutions [17].

When we study non extremal black holes in $A d S$ spacetimes we are no longer restricted to low energies, as we were in the discussion in higher dimensions [44,54]. The restriction came from matching the $A d S$ region to the Minkowski region. So the five dimensional results [53,54] can be used to describe arbitrary non-extremal black holes in three dimensional AntideSitter spacetimes. This might lead us to understand better where the degrees of freedom of black holes really are, as well as the meaning of the region behind the horizon. The question of the boundary conditions is very interesting and the conformal field theories should provide us with some definite boundary conditions and will probably explain us how to interpret physically spacetimes with horizons. It would be interesting to find the connection with the description of $2+1$ dimensional black holes proposed by Carlip [63].

In $[8,13]$ super-singleton representations of $A d S$ were studied and it was proposed that they would describe the dynamics of a brane "at the end of the world". It was also found that in maximally supersymmetric cases it reduces to a free field [8]. It is tempting therefore to identify the singleton with the center of mass degree of freedom of the branes [6,13]. A recent paper suggested that super-singletons would describe all the dynamics of $A d S$ [51]. The claim of the present paper is that all the dynamics of $A d S$ reduces to previously known conformal field theories.

It seems natural to study conformal field theories in Euclidean space and relate them to deSitter spacetimes.

Also it would be nice if these results could be extended to four-dimensional gauge theories with less supersymmetry.

\footnotetext{
${ }^{14}$ This possibility was also raised by [62], though it is a bit disturbing to find a constant energy flux to the UV (that is how we are interpreting the radial dimension).
} 


\section{Acknowledgments}

I thank specially G. Horowitz and A. Strominger for many discussions. I also thank R. Gopakumar, R. Kallosh, A. Polyakov, C. Vafa and E. Witten for discussions at various stages in this project. My apologies to everybody I did not cite in the previous version of this paper. I thank the authors of [64] for pointing out a sign error.

This work was supported in part by DOE grant DE-FG02-96ER40559.

\section{Appendix}

$D=p+2$-dimensional anti-deSitter spacetimes can be obtained by taking the hyperboloid

$$
-X_{-1}^{2}-X_{0}^{2}+X_{1}^{2}+\cdots+X_{p}^{2}+X_{p+1}^{2}=-R^{2},
$$

embedded in a flat $\mathrm{D}+1$ dimensional spacetime with the metric $\eta=\operatorname{Diag}(-1,-1,1, \cdots, 1)$. We will call $R$ the "radius" of $A d S$ spacetime. The symmetry group $S O(2, D-1)=S O(2, p+1)$ is obvious in this description. In order to make contact with the previously presented form of the metric let us define the coordinates

$$
\begin{aligned}
U & =\left(X_{-1}+X_{p+1}\right) \\
x_{\alpha} & =\frac{X_{\alpha} R}{U} \quad \alpha=0,1, \cdots, p \\
V & =\left(X_{-1}-X_{p+1}\right)=\frac{x^{2} U}{R^{2}}+\frac{R^{2}}{U} .
\end{aligned}
$$

The induced metric on the hyperboloid (A.1) becomes

$$
d s^{2}=\frac{U^{2}}{R^{2}} d x^{2}+R^{2} \frac{d U^{2}}{U^{2}} .
$$

This is the form of the metric used in the text. We could also define $\tilde{U}=$ $U / R^{2}$ so that metric (A.3) has an overall factor of $R^{2}$, making it clear that $R$ is the overall scale of the metric. The region outside the horizon corresponds to $U>0$, which is only a part of (A.1). It would be interesting to understand what the other regions in the $A d S$ spacetime correspond to. For further discussion see [65].

\section{References}

[1] J. Maldacena and A. Strominger, hep-th/9710014. 
[2] N. Seiberg, Phys. Lett. B408 (1997) 98.

[3] R. Haag, J. Lopuszanski, and M. Sohnius, Nucl. Phys. B88 (1975) 257.

[4] W.. Nahm, Nucl. Phys. B135 (1978) 149.

[5] G. Gibbons, Nucl. Phys. B207 (1982) 337; R. Kallosh and A. Peet, Phys. Rev. D46 (1992) 5223; S. Ferrara, G. Gibbons, R. Kallosh, Nucl. Phys. B500 (1997) 75.

[6] G. Gibbons and P. Townsend, Phys. Rev. Lett. 71 (1993) 5223.

[7] A. Salam and E. Sezgin, "Supergravities in Diverse Dimensions", Vol. 1 and 2, North-Holland 1989. Look for "gauged" supergravities.

[8] C. Frondal, Phys. Rev. D26 (1988) 82; D. Freedman and H. Nicolai, Nucl. Phys. B237 (1984) 342; K. Pilch, P. van Nieuwenhuizen and P. Townsend, Nucl. Phys. B242 (1984) 377; M. Günaydin, P. van Nieuwenhuizen and N. Warner, Nucl. Phys. B255 (1985) 63; M. Günaydin and N. Warner, Nucl. Phys. B272 (1986) 99; M. Günaydin, N. Nilsson, G. Sierra and P. Townsend, Phys. Lett. B176 (1986) 45; E. Bergshoeff, A. Salam, E. Sezgin and Y. Tanii, Phys. Lett. B205 (1988) 237; Nucl. Phys. D305 (1988) 496; E. Bergshoeff, M. Duff, C. Pope and E. Sezgin, Phys. Lett. B224 (1989) 71.

[9] M. Günaydin and N. Marcus, Class. Quant. Grav. 2 (1985) L11; Class. Quant. Grav. 2 (1985) L19; H. Kim, L. Romans and P. van Nieuwenhuizen, Phys. Lett. B143 (1984) 103; M. Günaydin, L. Romans and N. Warner, Phys. Lett. B154 (1985) 268; Phys. Lett. B164 (1985) 309; Nucl. Phys. B272 (1986) 598.

[10] M. Blencowe and M. Duff, Phys. Lett. B203 (1988) 229; Nucl. Phys. B310 (1988) 387.

[11] H. Nicolai, E. Sezgin and Y. Tanii, Nucl. Phys. B305 (1988) 483.

[12] M. Duff, G. Gibbons and P. Townsend, hep-th/9405124.

[13] P. Claus, R. Kallosh and A. van Proeyen, hep-th/9711161.

[14] G.. Horowitz and A. Strominger, Nucl. Phys. B360 (1991) 197.

[15] G. Gibbons, G. Horowitz and P. Townsend, hep-th/9410073.

[16] L. Susskind, hep-th/9611164; O. Ganor, S. Ramgoolam and W. Taylor IV, Nucl. Phys. B492 (1997) 191.

[17] M. Douglas, J. Polchinski and A. Strominger, hep-th/9703031. 
[18] M. Aganagic, C. Popescu and J. Schwarz, Nucl. Phys. B495 (1997) 99, hep-th/9612080.

[19] M. Dine and N. Seiberg, Phys. Lett. B409 (1997) 239.

[20] K. Becker, M. Becker, J. Polchinski and A. Tseytlin, Phys. Rev. D56 (1997) 3174.

[21] A. Polyakov, hep-th/9711002.

[22] T. Banks, W. Fishler, I. Klebanov and L. Susskind, hep-th/9709091.

[23] S. Gubser, I. Klebanov and A. Peet, Phys. Rev. D54 (1996) 3915; A. Strominger, unpublised.

[24] I. Klebanov, Nucl. Phys. B496 (1997) 231.

[25] S. Gubser, I. Klebanov and A. Tseytlin, Nucl. Phys. B499 (1997) 217.

[26] S. Gubser and I. Klebanov, hep-th/9708005.

[27] C. Vafa, Nucl. Phys. B469 (1996) 403.

[28] I. Klebanov and A. Tseytlin, Nucl. Phys. B475 (1996) 164.

[29] A. Srominger, Phys. Lett. B383 (1996) 44.

[30] E. Witten, hep-ih/9507121; N. Seiberg and E. Witten, Nucl. Phys. B471 (1996) 121.

[31] J. Maldacena, Proceedings of Strings'97, hep-th/9709099.

[32] N. Seiberg, Phys. Rev. Lett. 79 (1997) 3577.

[33] A. Sen, hep-th/9709220.

[34] N. Seiberg, hep-th/9705117.

[35] S. Sethi and L. Susskind, Phys. Lett. B400 (1997) 265.

[36] T. Banks and N. Seiberg, Nucl. Phys. B497 (1997) 41.

[37] T. Banks, W. Fishler, N. Seiberg and L. Susskind, Phys. Lett. B408 (1997) 111.

[38] I. Bars, Phys. Rev. D55 (1997) 2373.

[39] A. Strominger and C. Vafa, Phys. Lett. B379 (1996) 99.

[40] G. Horowitz, J. Maldacena and A. Strominger, Phys. Lett. B383 (1996) 151. 
[41] L. Andrianopoli, R. D'Auria and S. Ferrara, Int. J. Mod. Phys $\mathbf{A 1 2}$ (1997) 3759.

[42] S. Ferrara, R. Kallosh and A. Strominger, Phys. Rev. D52 (1995) 5412; S. Ferrara and R. Kallosh, Phys. Rev. D54 (1996) 1514; Phys. Rev. D54 (1996) 1525.

[43] J. C. Breckenridge, R. C. Myers, A. W. Peet and C. Vafa, Phys. Lett. B391 (1997) 93.

[44] J. Maldacena, Phys. Rev. D55 (1997) 7645.

[45] D. Diaconescu and N. Seiberg, J. High Energy Phys. 7 (1997) 001.

[46] O. Aharony, M. Berkooz, S. Kachru, N. Seiberg and E. Silverstein, hepth/9707079.

[47] E. Witten, hep-th/9707093.

[48] Bañados, Teitelboim and Zanelli, Phys. Rev. Lett. 69 (1992) 1849.

[49] S. Hyun, hep-th/9704005.

[50] H. Boonstra, B. Peeters and K. Skenderis, hep-th/9706192

[51] K. Sfetsos and K. Skenderis, hep-th/9711138.

[52] S. Hellerman and J. Polchinski, hep-th/9711037.

[53] G. Horowitz and A. Strominger, Phys. Rev. Lett. 77 (1996) 2368, hepth/9602051.

[54] A. Dhar, G. Mandal and S. Wadia Phys. Lett. B388 (1996) 51; S. Das and S. Mathur, Nucl. Phys. B478 (1996) 561; Nucl. Phys. B482 (1996) 153; J. Maldacena and A. Strominger, Phys. Rev. D55 (1997) 861; S. Gubser, I. Klebanov, Nucl. Phys. B482 (1996) 173; C. Callan, Jr., S. Gubser, I. Klebanov and A. Tseytlin, Nucl. Phys. B489 (1997) 65; I. Klebanov and M. Krasnitz, Phys. Rev. D55 (1997) 3250; I. Klebanov, A. Rajaraman and A. Tseytlin, Nucl. Phys. B503 (1997) 157; S. Gubser, hep-th/9706100; H. Lee, Y. Myung and J. Kim, hep-th/9708099; K. Hosomichi, hep-th/9711072.

[55] D. Birmingham, I. Sachs and S. Sen, hep-th/9707188.

[56] M. Cvetic and F. Larsen, Phys. Rev. D56 (1997) 4994; hep-th/9706071.

[57] J. Maldacena, A. Strominger and E. Witten, hep-th/9711053. 
[58] A. Chamseddine, S. Ferrara, G. Gibbons and R Kallosh, Phys. Rev. D55 (1997) 3647.

[59] J. Maldacena and A. Strominger, Phys. Rev. D56 (1997) 4975.

[60] J. Maldacena and L. Susskind, Nucl. Phys. B475 (1996) 679.

[61] T. Banks, W. Fischler, S. Shenker and L. Susskind, hep-th/9610043.

[62] O. Aharony, S. Kachru, N. Seiberg and E. Silverstein, private communication.

[63] S. Carlip, Phys. Rev. D51 (1995) 632.

[64] R. Kallosh, J. Kumar and A. Rajaraman, hep-th/9712073.

[65] S. Hawking and J. Ellis, "The large scale structure of spacetime", Cambrige Univ. Press 1973, and references therein. 